\title{
Experiencias sobre el impacto del Programa de Formación en Ética de la Investigación Biomédica y Psicosocial en el ámbito de la salud mental y la investigación conductual
}

\author{
Liliana Mondragón Barrios ${ }^{1}$
}

\begin{abstract}
Resumen: El propósito de este trabajo es presentar el impacto y la integración que los conocimientos adquiridos en el Programa Internacional de Formación en Ética de la Investigación Biomédica y Psicosocial de la Universidad de Chile han tenido en mi experiencia profesional, en el ámbito de la investigación psicosocial en un Instituto de Salud de México. Para este objetivo, expondré tres áreas en las cuales se ha podido evidenciar tal impacto: trabajo en los comités de ética, desarrollo de programas de académicos en bioética e investigación y publicación sobre ética y bioética. El motivo que me llevó a incursionar en el Programa fue que su enseñanza vincula la investigación psicosocial con la ética y la bioética, lo cual me permitió dirigir este tipo de reflexión hacia problemas como violencia, suicidio, adicciones, depresión y salud mental, y a nuevos campos como los estudios comunitarios, con poblaciones en riesgo o vulnerables, en los cuales las diversas implicaciones son difíciles de indagar.
\end{abstract}

Palabras clave: ética de la investigación, bioética, ciencias de la conducta, salud mental, investigación conductual, intento de suicidio, psicología social

\section{Experiences about the impact of the Ethics of Biomedical and Psychosocial Research Training Program in mental health and behavioral research field}

\begin{abstract}
The purpose of this article is to present the impact and the integration of knowledge acquired in the international ethics of biomedical and psychosocial research training program of the University of Chile has had in my professional experience in the field of psychosocial research at a Mental Health Institute in Mexico. With this purpose, I will develop three areas of clear impact: participation in scientific ethical review committees, development of academic programs in bioethics and research and publication on ethics and bioethics. My motivation to enter in the program was that this training links psychosocial research with ethics and bioethics, which allows me to apply this type of reflection to problems such as violence, suicide, addictions, depression and mental health, and new fields such as community studies with vulnerable or at risk populations, in which the diverse implications are difficult to inquire.
\end{abstract}

Key words: ethics of research, bioethics, behavioral sciences, mental health, behavioral research, suicidal attempt, social psychology

\section{Experiências sobre o impacto do Programa de Formaçáo em Ética da Pesquisa Biomédica e Psicossocial no âmbito da saúde mental e pesquisa comportamental}

Resumo: O propósito deste trabalho é apresentar o impacto e a integração que os conhecimentos adquiridos no Programa Internacional de Formação em Ética da Pesquisa Biomédica e Psicossocial da Universidade do Chile tiveram em minha experiência profissional no âmbito da pesquisa psicossocial no Instituto de Saúde do México. Para este objetivo, mostrarei três áreas nas quais foi possível evidenciar tal impacto: trabalho nos comitês de ética, desenvolvimento de programas acadêmicos em bioética e pesquisa e publicação sobre ética e bioética. O motivo que me levou a incursionar no Programa foi que o seu aprendizado vincula a pesquisa psicossocial com a ética e a bioética, o que me permitiu dirigir este tipo de reflexão para problemas como violência, suicídio, toxicofilias, depressão e saúde mental, e a novos campos como os estudos comunitários com populações em risco ou vulneráveis, nas quais as diversas implicaçôes são difíceis de indagar.

Palavras-chave: ética da pesquisa, bioética, ciências do comportamento, saúde mental, pesquisa comportamental, intenção de suicídio, psicologia social

\footnotetext{
${ }^{1}$ Doctora en Psicología. Instituto Nacional de Psiquiatría Ramón de la Fuente Muñiz, México. Becaria del Programa de Ética de la Investigación Biomédica y Psicosocial, Fogarty International Center, Grant R25 TW006065, 2005.

Correspondencia: lilian@imp.edu.mx
} 


\section{Introducción}

El Programa Internacional de Formación en Ética de la Investigación Biomédica y Psicosocial, del Centro Interdisciplinario de Estudios en Bioética (CIEB) de la Universidad de Chile, tiene como objetivo ofrecer a calificados profesionales de América Latina y el Caribe una formación multidisciplinaria en ética de la investigación en seres humanos. Asimismo, promueve el desarrollo de capacidades, la conciencia pública y la toma de decisiones referidas a la ética de la investigación biomédica y conductual(1). Este último tipo de investigación cobra mayor interés por la escasa información de los aspectos éticos aplicados en este campo y porque pocos programas de entrenamientos en ética suelen abordarlo.

El motivo que me llevó a incursionar en el Programa del CIEB fue porque en su enseñanza vincula la investigación psicosocial, ámbito en el que me desarrollo profesionalmente, con la ética y la bioética. Ello me permitió dirigir este tipo de reflexión hacia problemas como violencia, suicidio, adicciones, depresión, salud mental, y a nuevos campos de acción, por ejemplo, estudios comunitarios con poblaciones en riesgo o vulnerables, en las cuales las diversas implicaciones son difíciles de indagar(2).

La finalidad de mi capacitación y formación en el campo ético ha sido para dar respuesta, subsanar o mediar, con argumentos y conocimientos sólidos, razonados y fundamentados, las nuevas problemáticas en los diferentes campos de la investigación conductual. No se trata de reducir el terreno complejo de las acciones morales a una visión psicológica, sino de incluir una disertación en torno a la contingencia de la condición y no circunscribirla a situaciones ideales de comportamiento(3).

El propósito de este trabajo es presentar el impacto y la integración de los conocimientos adquiridos en el Programa Internacional de Formación en Ética de la Investigación Biomédica y Psicosocial en mi experiencia profesional, dentro del ámbito de la investigación psicosocial, en un Instituto de Salud en México. Para este objetivo, expondré tres áreas en las cuales se ha podido evi- denciar dicho impacto: 1) trabajo en los comités de ética, 2) desarrollo de programas de académicos en bioética y 3) investigación y publicación sobre ética y bioética.

\section{Trabajo en los comités de ética}

Los comités de ética se encuentran entre las manifestaciones más visibles de la bioética en el ámbito social, orientado a las reformas y al trabajo multidisciplinario(3). En estas instancias se pone en práctica el proceso deliberativo como elemento fundamental, enmarcado por el diálogo plural que permite establecer la creación de un consenso institucional.

La educación teórica y práctica acerca de los comités de evaluación ética proporcionada por el Programa forma a sus egresados como expertos competentes en esta materia, pero principalmente como agentes de cambio para cualquier institución(4). Este adiestramiento ha repercutido en $\mathrm{mi}$ desempeño como miembro de cuatro comités de instituciones, tanto de salud como educativas en México.

Únicamente la preparación formal y constante en el área de la ética en la investigación, va forjando la capacidad de analizar y emitir argumentos orientados a identificar el curso de acción ético más razonable o válido de los proyectos de investigación, con el objeto de garantizar el bienestar y los derechos de los participantes(3).

Entre los ejemplos del impacto de la conjunción de mi profesión como psicóloga social y la capacitación en bioética, respecto del trabajo al interior de algunos comités de ética en investigación, resaltan dos momentos: en primer lugar, mi participación en la creación de un comité para la evaluación de estudios en el área de las adicciones a drogas y/o alcohol. La investigación psicosocial en adicciones, representa un campo fértil; sin embargo, los riesgos de reproducir el estigma y la discriminación que acompaña a los usuarios de drogas son altos si no se cavila y se sobreponen las consideraciones éticas. El respeto a la confidencialidad y privacidad, la protección de la integridad del usuario en el contexto sociocultu- 
ral, incluyendo al propio investigador, etc., son requerimientos que deben contemplarse en esta área. Tampoco pueden dejarse a un lado las implicaciones históricas, jurídicas y políticas de las adicciones, que tienen una relación estrecha con la ética y que, como en ningún otro campo de estudio, se pueden dilucidar.

El segundo momento fue la consultoría relativa a la normativa ética y legal para un comité de ética en investigación de una institución de salud. Este comité dictamina anualmente alrededor de 200 protocolos de investigación biomédica y psicosocial, algunos en colaboración con otros países. Por esta razón, era necesario el registro del comité ante el Institutional Review Board (IRB), del U.S. Department of Health and Human Services (DHHS), la Office of the Human Research Protections (OHRP) y la aprobación del Federalwide Assurance (FWA) for the Protection of Human Subjects. Ambos se consiguieron en enero de 2008. Posteriormente, en 2010, me invitarían a formar parte de dicho comité, en el cual, además de participar en el trabajo deliberativo, formalizaría, reestructuraría y optimizaría su funcionamiento.

La capacitación sobre diversas disciplinas vinculadas al diálogo bioético que ofrece el Programa, como Antropología, Derecho, Medicina, Filosofía, permite una evaluación ética más profunda y exhaustiva de los protocolos de investigación. Por ejemplo, para la revisión de un proyecto científico se requiere también conocer la legislación nacional en materia de investigación, además de pautas y declaraciones internacionales sobre ética o derechos humanos. Asimismo, es importante tomar en cuenta el contexto social y cultural de los participantes o argumentar los aspectos éticos en un dictamen no satisfactorio de un estudio evaluado.

La educación formal en el ámbito de la ética tiene repercusiones evidentes en la labor de los comités. Primero, definitivamente nos aleja de la aplicación rutinaria de principios generales o de normas morales aceptadas. Segundo, el proceso deliberativo es el elemento fundamental del análisis, lo que permite establecer "garantías mínimas para identificar los aspectos relevantes por considerar, de modo que la acción práctica, en de- terminadas circunstancias y en casos complejos, responda a los valores en cuestión"(3:15), y que estos den contenido a los principios éticos. Tercero, reflexión y crítica son dos capacidades que se van desarrollando como herramientas básicas de la actividad creativa en estos grupos colegiados, incluso para detectar deficiencias o factores que obstaculizan su funcionamiento o fortalecimiento.

En algunas instituciones de salud y académicas en México, el trabajo de los comités de ética en investigación sigue siendo un campo desestimado, al percibirse como un requisito burocrático que afecta los tiempos de la investigación. La falta de interés y conocimiento del ejercicio ético en el campo científico es el principal obstáculo para constituir una cultura ética en el trabajo investigativo, pero sobre todo para otorgar garantía pública, es decir, una construcción más fiable de la conciencia bioética institucional.

Lo anterior abre la posibilidad de continuar trabajando en el terreno fructífero de los comités de evaluación ética, con el fin de consolidar la función que tienen como creadores de consensos, encomienda asumida por agentes responsables y capacitados en ética y bioética.

\section{Desarrollo de programas de académicos en bioética}

Mi formación en bioética tiene en sí misma impacto en la capacitación y especialización de los miembros de la institución de salud donde laboro, y es que esta institución siempre ha estado interesada en la formación continua de sus investigadores, porque comprende que ello suscita a su vez un efecto benéfico sobre la institución misma. Gracias a este interés y apoyo institucional, y a que fui seleccionada por el CIEB, pude participar como becaria del Programa Internacional de Formación en Ética de la Investigación Biomédica y Psicosocial de la Universidad de Chile/OPSOMS, Fogarty Internacional Center, 2005-2006.

El retorno a mi institución de pertenencia, después del curso de formación en ética, creó de al- 
guna manera la posibilidad de aplicar los saberes "novedosos" en el ámbito de la salud mental y la investigación psicosocial, principalmente. Sin embargo, como todo lo nuevo, este aprendizaje trajo consigo también reticencia por parte de los profesionales de la salud de la institución.

Uno de los criterios y requisitos del CIEB para participar en el Programa es que los candidatos pertenezcan a una institución en su país de origen, que garantice la reincorporación del alumno al trabajo institucional, con el fin de que pueda emprender acciones o actividades basadas en el conocimiento ético que fortalezcan el quehacer de la institución(1). A pesar de que este precepto tiene la intención de que el candidato retribuya en servicios lo que ha recibido en formación y que su institución se comprometa a facilitar la realización de estos para dar continuidad a la encomienda ética, algunas instituciones no son tan receptivas para la expresión de la conciencia crítica y reflexiva del saber ético; por ello se necesita una gestión previa: la sensibilización.

En mi experiencia, el trabajo de sensibilización ha consistido en difundir información comprensible y concreta de lo que es la bioética y su vinculación práctica en determinado campo de acción, en conferencias o clases breves, incluso en pláticas informales. Dicha labor no solo se dio al regreso de la capacitación, sino que se ha prolongado hasta hoy.

Casi cuatro años más tarde de mi reintegración laboral y de la sensibilización, tuve la oportunidad de proponer y plantear cursos y seminarios de educación continua, más extensos y formales, sobre temas de bioética y ética en investigación. Por ejemplo, a partir de 2010 se abrió el curso anual "La bioética en la salud mental”, cuyo propósito es introducir el análisis de los aspectos bioéticos implicados en los distintos escenarios del campo de la salud mental, con el fin de que su comprensión y pericia sea incorporada al ejercicio profesional en este ámbito. La coordinación de este curso me ha permitido invitar y colaborar con investigadores de otras instituciones nacionales prestigiosas, incluyendo a colegas egresados también del Programa del CIEB.
Otra propuesta fue impartir un seminario sobre ética en la investigación psicosocial, debido al interés por mi formación como psicóloga social y a que este tema es poco abordado. El objetivo del seminario fue analizar y debatir los problemas sustanciales de las prácticas de investigación; en él participaron investigadores consolidados y estudiantes, y tuvo una duración de cinco meses con un total de 25 horas.

Diferentes medios de enseñanza no presenciales, como las videoconferencias, se han utilizado para difundir el quehacer de la bioética y salud mental, con el propósito de abarcar mayor audiencia en diversas instituciones de salud y educación, en México y otros países. La videoconferencia ha tenido la intención de revisar los aspectos bioéticos en circunstancias y situaciones problemáticas que encierra el ejercicio profesional en el campo de la salud mental, y que pueden convertirse en dilemas éticos.

Lo antes mencionado es un breve panorama del liderazgo activo en la enseñanza de la bioética, con base en la formación y capacitación en dicha materia, lo que a su vez responde, por un lado, a las necesidades y misión de un instituto nacional de salud en México y, por otro, al compromiso con el Programa del CIEB de apoyar y crear estos campos dentro de la institución de pertenencia.

En otro sentido, el Programa permite una personalización de los contenidos curriculares de acuerdo con los intereses profesionales específicos de cada participante(1). En mi caso, los componentes de la ética en la investigación psicosocial que se ofrecían se conjugaban adecuadamente con mi trabajo profesional. Por esta razón, el desarrollo de acciones académicas, como cursos, seminarios, entre otros, se centra en el tema de la salud mental y la investigación de tipo conductual y social.

Tanto la salud mental como la investigación psicosocial son áreas que han estado escasamente vinculadas a las preocupaciones éticas, en cuanto a su aplicación cotidiana en los diversos escenarios que ocupan. Esto ha representado en mi vivencia una serie de obstáculos para insertar o introducir el saber o diálogo ético en estos campos, debido 
a que, en ocasiones, impera más un discurso con "creencias" morales, es decir, más dóxico(5) que epistémico(6).

No obstante lo anterior, he colaborado impartiendo seminarios que pertenecen a la curricula de posgrados de dos instituciones públicas de educación superior en México. La maestría y el doctorado en salud mental pública que ofrece conjuntamente la Facultad de Medicina de la Universidad Nacional Autónoma de México (UNAM) y el Instituto Nacional de Psiquiatría Ramón de la Fuente, dentro del Programa de Posgrado en Ciencias Médicas, Odontológicas y de la Salud; y la maestría en ciencias en bioética del Instituto Politécnico $\mathrm{Na}$ cional (IPN).

La docencia en las principales instituciones educativas de México me ha permitido ampliar mi colaboración con otros profesionales interesados en la bioética, con diferentes perspectivas en esta área, lo cual ha enriquecido la labor de enseñanza. Cabe resaltar que, cuando me integro a estos grupos de trabajo, el sello distintivo de mi formación en ética suele ser lo conciernente a ética en investigación psicosocial, no solo por mi formación e interés, sino porque, como mencioné, aún hay grandes vacíos respecto de esta materia.

El trabajo de los alumnos de posgrado en bioética marca una cierta visión hacia el futuro del quehacer en este campo. Temas como adicciones o paradigma emergente son nuevas narrativas y retos en el discurso tradicional de la bioética. Como acompañante y testigo de las inquietudes científicas de los estudiantes, mi función es proveer de herramientas, habilidades y hasta actitudes para comprender los saberes que la bioética demanda.

Estudiantes ahora y colaboradores después, el interés por la bioética como disciplina a académica se ha incrementado entre los profesionales de salud de mi institución. Al menos tres o cuatro están inscritos en posgrados de bioética. No es fácil abrir el camino para los que vienen, pero es necesario, para que se multiplique el diálogo.

En la cuestión de capacitación es muy importante impartir cursos, seminarios y talleres, pero de igual forma es imprescindible asistir a estos eventos. La formación en cualquier ámbito debe actualizarse de manera continua. En muchas ocasiones he acudido a los eventos científicos y académicos, tanto nacionales como internacionales, entre los que se incluyen los ofrecidos por el CIEB.

\section{Investigación y publicación sobre ética y bio- ética}

Uno de los muchos objetivos del Programa del CIEB es que sus egresados sean capaces de asumir un rol activo en la planificación y conducción de investigación biomédica, conductual y epidemiológica, con base en los principios bioéticos(1).

Como investigadora he desarrollado diversos estudios sobre la conducta suicida desde distintas perspectivas —epidemiológica, filosófica, psicológica, psicoanalítica-, los cuales me permitieron entender que gran parte de la problemática suicida plantea dilemas éticos difíciles de dilucidar. Este interés me llevó a proponer una investigación sobre disyuntivas en las concepciones bioéticas que afectan la terapéutica del intento suicida, como tesis doctoral en psicología social, en la Universidad Nacional Autónoma de México (UNAM) $(7,8)$. Sin embargo, para elaborar dicho estudio requería una enseñanza formal en la disciplina bioética que ofreciera contenidos afines a mi profesión y que pudiera fortalecer mi ejercicio profesional en la investigación psicosocial.

La formación en ética que me brindó el CIEB me concedió el impulso no solo para concluir mi trabajo doctoral, sino para proponer nuevas líneas de investigación en mi institución, como bioética y salud mental, y ética en investigación psicosocial. La investigación en la aplicación de los principios bioéticos en la atención clínica y la prevención del intento de suicidio fue el primer estudio bioético registrado en mi institución y en el que participaron como colaboradores algunos profesores del CIEB. Asimismo, este estudio fue financiado por el Consejo Nacional de Ciencia y Tecnología (CONACYT) de México (91082), del 15 de diciembre de 2008 al 30 de abril de 2011. 
El siguiente proyecto de investigación aborda los componentes éticos en la investigación social en salud mental, esto es, la preocupación por los retos que plantean fenómenos como la violencia, las adicciones, la depresión o el suicidio - entendiendo que deben incluir aspectos éticos particulares de este tipo de investigación-, con el fin de instrumentar los mecanismos que faciliten el desarrollo de la ciencia social en salud basada en principios éticos. Este es un estudio colaborativo con profesores del CIEB. De igual forma está financiado por Consejo Nacional de Ciencia y Tecnología (CONACYT) de México (169226).

La conducción de investigaciones sobre bioética y ética en salud mental e investigación psicosocial ha evidenciado el impacto que tuvo mi capacitación en el CIEB. Al mismo tiempo se ha creado una red de colaboración para el desarrollo de estas pesquisas entre este centro y el instituto de salud donde laboro. Dicha red también es un objetivo planteado por el Programa de Formación en Ética, por lo que en el plano del tema de la investigación se cumplen dos importantes objetivos que el Programa espera de sus egresados: por un lado, el mando activo en la investigación y, por otro, la creación de redes de cooperación entre bioeticistas.

En cuanto a la difusión de los resultados de los estudios realizados en materia de bioética y salud mental, se han expuesto con buena recepción en foros nacionales e internacionales. Nuevamente, esta asociación poco común entre el abordaje ético y los problemas como conducta suicida, adiciones, violencia, entre otros, tienen una atención especial en cualquier evento de bioética, en los cuales, por lo general, se exponen cuestiones de biomedicina.

Otra forma de difundir los hallazgos de las investigaciones realizadas en el campo que nos ocupa son las publicaciones científicas y otros textos de divulgación o de orden normativo, como políticas, directrices, pautas o guías. En este rublo, el Programa plantea que su egresados estén en condiciones de prever el impacto social de la investigación en la perspectiva de formular políticas públicas — de salud y otros sectores claves-, orientadas a mejorar la calidad de vida y reducir las inequidades en el acceso a los servicios de atención de salud y en el estado de salud(1).

Si bien los resultados de las investigaciones científicas aspiran a convertirse en políticas públicas, o al menos a tener repercusión en ellas, lo cierto es que no siempre sucede esto. Lo que sí es posible es la creación de documentos que intenten contribuir o generar una optimización en la atención clínica de los pacientes, incluyendo a los enfermos mentales. Por ejemplo, reduciendo el estigma o la discriminación, o promoviendo la equidad y los derechos humanos.

En mi experiencia, quizá el escrito más relevante fue la elaboración de la "Guía bioética para el tratamiento clínico de pacientes que han intentado suicidarse" (9). En este documento participó un equipo transdisciplinario de expertos en bioética, nacionales e internacionales, entre ellos profesores del CIEB. La Guía ofrece recomendaciones bioéticas para el buen ejercicio de la práctica clínica, con lo cual pretende colaborar en la prevención de un nuevo intento o, más aún, de la consumación de un suicidio. Asimismo, facilita al profesional de la salud la aplicación de los principios bioéticos (beneficencia, autonomía y justicia) en las intervenciones terapéuticas con personas en riesgo de intento suicida, con base en el respeto de los derechos de los pacientes (trato digno, respetuoso, confidencial, etc.), con el fin de evitar dilemas éticos. Además, puede ser utilizada como programa de capacitación, consulta u orientación en los escenarios clínicos y en la formación académica de los especialistas en salud mental.

La Guía fue financiada por el Consejo Nacional de Ciencia y Tecnología (CONACYT) y presentada por el Instituto Nacional de Psiquiatría Ramón de la Fuente Muñiz como un aporte novedoso en el campo de la bioética y la salud mental.

En cuanto a los artículos científicos, el tema de mayor recurrencia en mis manuscritos ha sido la ética en la investigación psicosocial y los apuntes relacionados en torno a su reflexión en las ciencias sociales y en el campo de la salud. Estos textos han sido publicados en revistas indizadas, con 
alto factor de impacto, y en capítulos de libros. Esta área es un campo fecundo para fortalecer y diseminar el conocimiento de la bioética.

\section{Reflexiones finales: perspectivas}

De acuerdo con el Programa Internacional de Formación en Ética de la Investigación Biomédica y Psicosocial, se espera que, a su término, los participantes estén capacitados para asumir un liderazgo activo en la enseñanza, evaluación, diseño y conducción de investigaciones éticamente sustentables, colaborar en normativas y regulaciones de ética de la investigación, así como participar en comités de evaluación ética de la investigación científica, con el compromiso de apoyar y crear estos campos dentro de su institución(1). Bajo dicha misión, mi compromiso como egresada del Programa de ética es continuar reforzando los mecanismos que consoliden un marco ético, que trascienda a cada una de las tareas encomendadas a la comunidad de mi institución. En resumen, se trata de:

- Fomentar la capacitación de la comunidad científica en temas relacionados con la ética de la investigación, a través de seminarios y cursos.
- Elaborar guías bioéticas en temas inherentes a los conflictos en investigación y en respuesta a las necesidades de esta.

- Fortalecer líneas de investigación sobre ética y bioética.

- Formar recursos humanos en el ámbito de la ética de la investigación.

- Publicar artículos de investigación científica de alto impacto sobre los resultados de las investigaciones en este campo.

- Formalizar grupos de discusión sobre dilemas éticos en la investigación con los actores participantes.

- Consolidar a los comités de ética en los que participo, como instancia de diálogo y transparencia en el campo de la investigación en neurociencias, clínica, epidemiológica y social.

\section{Agradecimientos}

Este trabajo fue posible gracias al proyecto "La aplicación de los principios bioéticos en la atención clínica y la prevención del intento de suicidio", del Instituto Nacional de Psiquiatría Ramón de la Fuente INPRFM-4601. Financiamiento por el Consejo Nacional de Ciencia y Tecnología (CONACyT-91082). 


\section{Referencias}

1. Centro Interdisciplinario de Estudios en Bioética (CIEB). Programa Avanzado de Formación en Ética de la Investigación Biomédica y Psicosocial. Universidad de Chile. Disponible en http://www. bioetica.uchile.cl/cursos/postulacion.htm

2. Mondragón L. Ética de la investigación psicosocial. Salud Mental 2007; 30: 25-31.

3. Comisión Nacional de Bioética. Guía Nacional para la Integración y Funcionamiento de Comités de Ética en Investigación. México: Comisión Nacional de Bioética, Secretaría de Salud; 2010.

4. Moreno J. Ethics Committee and Ethics Consultants. In Kuhse H, Singer P, (eds.) A Companion to bioethics. 2nd ed. Singapur: Wiley-Blackwell, 2009: 573-583.

5. Ferrater Mora J. Diccionario de Filosofía. Barcelona: Editorial Ariel; 1999.

6. Álvarez J, Lolas F, Outomuro D. Historia de la ética en investigación con seres humanos. En Lolas F, Quezada A, Rodríguez E, (eds.) Investigación en Salud. Dimensión Ética. Santiago de Chile: Centro Interdisciplinario de Estudios en Bioética, Universidad de Chile, 2006: 39-46.

7. Mondragón L. El suicidio ¿el derecho a morir?Tesis para obtener el grado de Doctora en Psicología. México: Programa de Maestría y Doctorado en Psicología. Universidad Nacional Autónoma de México, 2008.

8. Mondragón L, Monroy Z, Ito MA, Medina-Mora ME. Disyuntivas en las concepciones sobre autonomía y beneficencia que afectan la terapéutica del intento suicida. Acta Bioethica 2010; 16(1): 77-86.

9. Mondragón L, Ito ME, Monroy Z, Netza C, Ocampo R, Rodríguez E, et al. Guía bioética para el tratamiento clínico de pacientes que han intentado suicidarse. México: Instituto Nacional de Psiquiatría Ramón de la Fuente Muñiz, Secretaría de Salud; 2010.

Recibido: 1 de marzo de 2012

Aceptado: 18 de marzo de 2012 